\title{
A Comparative Performance Analysis of 6to4 Transition Mechanism over MPLS and Frame Relay using OPNET
}

\author{
Oliver Kufuor Boansi \\ Department of Information Technology Education \\ University of Education, Winneba \\ Winneba, Ghana
}

\begin{abstract}
IPv6 addressing is taking over from IPv4 with the exhaustion of the latter's address space. However, both versions of IP are incompatible hence the need for transition technologies to ensure their coexistence. This research analyzed the performance of the 6to4 transition mechanism when it ran over two separate WAN technologies namely MPLS and Frame Relay by way of simulating the scenarios using OPNET 14.5. Traffic generated for this analysis were from FTP, Email and Database query, and were used to measure and analyze performance metrics such as end-to-end delay, response time and point to point link utilization over an hour of simulation time to ascertain which WAN and transition mechanism provided the best performance. The simulation values at the end of the research indicated that 6 to 4 together with MPLS produced lower values for all the performance metrics studied.
\end{abstract}

\section{General Terms}

Computer Networking, Simulation

\section{Keywords}

Transition Mechanisms, 6to4, MPLS, Frame Relay, OPNET, WAN, IPv4, IPv6.

\section{INTRODUCTION}

The advancement in the usage of computers and other devices on the internet has ensured that address space of the IPv4 are exhausted as published by Number Resource Organization [1]. The increase in computers is not the only reason for exhaustion of address space but also the poor management on the usage of address blocks allocated to some users. As a result of the exhausted address space, IPv6 has been created with a larger address space to make provision for every device and other internet enabled objects on the surface of the earth. The deployment of a full IPv6 network has been delayed though it was expected to achieve a faster deployment rate in the midst of an exhausted IPv4 address space. This slow deployment has largely been attributed to the cost involved in investing in a complete IPV6 devices and equipment [2]. APNIC Lab reports the worldwide deployment rate as $5 \%$ as at July 2015.The process of switching from IPv4 to an entirely IPV6 network will take very long to be achieved as these two protocols are also not backward compatible [3]. As a result, there are other mechanisms that have been put in place to ensure that they can coexist until a full IPv6 network is realized. These mechanisms include translation, tunneling and dual stack. Two common Wide Area Networks (WAN) utilized are Multiprotocol Label Switch (MPLS) and Frame Relay (FR). This research compared and analyzed how the 6to4 transition mechanism performed when it was configured on an MPLS and then on FR infrastructure. 6to4 is an example of a tunneling transition mechanism.

\author{
Michael Asante, PhD \\ Department of Computer Science \\ Kwame Nkrumah University of Science and \\ Technology \\ Kumasi, Ghana
}

\section{LITERATURE REVIEW AND RELATED WORKS}

Babar et.al [4] examined several tunneling mechanisms of IPv6 transition deployed on MPLS to evaluate and compared their performances. They observed that ISATAP has a better performance in all metrics used.

Suhail, Wajid \& Mohsin [5] evaluated the performance of IPV6 and IPV4 over MPLS network. They concluded that, the delay, voice jitter for IPV6 over MPLS was higher than that of IPV4 over MPLS. They also observed that IPV6 over MPLS performed better than IPV4 over MPLS in terms of throughput and link utilization

Grayeli, Sarkani \& Mazzuchi [6] analyzed the performance of IPV6 transition mechanisms over MPLS. The mechanisms under study included GRE, Automatic IPV4-compatible tunnel, 6to4 and dual stack. These were done between Customer Edge (CE) routers and also between Provider Edge $(\mathrm{PE})$ routers. It emerged that dual stack had the best overall performance metrics with the lowest delay, jitter and highest throughput.

Rani and Vandana [7] in a paper evaluated the performance of IPV6 transition technologies. They had networks that separately had dual stack, 6to4 and manual tunnel, IPV4 and IPV6 configured. The network metrics used in measuring their performance included CPU utilization, delay and network throughput. It emerged that CPU utilization for manual tunnel and 6to4 were greater as compared to the others understudy. Network delay was low for dual stack but were higher for 6to4 and manual. The network throughput results showed that IPV6 was better than the others compared.

Khannah \& Alsadeh [8] evaluated the impact of IPv4/IPv6 transition techniques such as dual stack, automatic tunneling and manual tunneling on some applications. They found varied results among the techniques. Dual stack performed better than tunneling in terms of response time. However, for throughput and jitter, tunneling outperformed dual stack.

Albkerat and Isaac [9] performed an analytical study on IPv6 transition mechanisms namely dual stack, tunnel and translation to demonstrate how these mechanisms affect network behavior.

In their work [10], empirically analyzed the feasibility of IPv6 transition technologies in relation to specific network scenarios. $\mathrm{Wu}$ and Zhou [11] analyzed and tested three transition mechanisms (dual stack, ISATAP tunnel and 6to4 tunnel) and found out that there are some performance advantages on dual stack protocol mechanism IPv6 network than IPv4, IPv6 ISATAP tunnel and IPv6 6to4 tunnel network. 
Hossain et.al [12] in their study deployed and analyzed in a controlled environment, three transition mechanisms (Dual stack, 6to4 tunneling and NAT-PT) using the packet tracer simulator. They concluded that 6to4 tunneling method produced the best results in terms of latency, throughput and packet loss whereas NAT-PT produced the worst results among the three mechanisms under study. Kalwar, Bohra \& Memon [13] identified that Dual Stack transition mechanism allowed a seamless transitioning of packets from IPv4 to IPv6 networks simultaneously based on a test bed utilized.

Singalar \& Banakar [14] undertook a performance analysis of IPv4 and IPv6 transition mechanisms and found out that dual stack and tunneling were at par and described them both as suitable for transitioning from IPv4 to IPv6. However, they recommended dual stack over tunneling because of the two transition mechanisms, dual stack had the better performance. NAT-PT was not recommended because of its poor latency.

Kim [15] analyzed and compared tunneling based IPv6 transition mechanisms from a variety of views.

\section{TUNNELLING TRANSITION MECHANISMS}

Tunneling provides a virtual channel between networks with heterogeneous versioned addresses so that they can transmit packets as though they come from the same versions of IP addresses. This is achieved by placing IPv6 packets into IPv4 packets and then routed through an IPv4 router. 6to4 [16], 4over6 [17], 6in4 [18] and public 4over6 [19] are some examples of tunneling mechanism.

\subsection{6 to4 TRANSITION MECHANISMS}

This mechanism allows for the connection IPv6 sites that have been isolated by IPv4 network. The IPv4 network normally acts as the link between these isolated networks. The IPv4 could be a service provider as it is the case in most instances and are needed to be able to connect these networks mostly located at different geographical sites. There are no explicit tunnel definitions for this type of transition mechanism. IPv6 packets are encapsulated in IPv4 packets and transmitted over IPv4 network. Upon reaching the destination 6to4 router, the packet is de-encapsulated. RFC 3056 [16] throws more light on this tunnel technology.

\section{RESEARCH PROBLEM}

IPv4 and IPv6 as already is common knowledge, are not compatible. Transition mechanisms has been put in place to ensure that this incompatibility issue is rectified or accommodated. The challenge is to determine the performance of these transition mechanisms on the various WAN (MPLS and FR) technologies.

According to [20], performance of networks has an impact on every business. Research into learning about new technologies and how they are integrated into existing ones and their impact on these existing networks is important as they may tend to affect a network's performance positively or negatively.

\section{METHODOLOGY}

In order to achieve the aim and objectives of the study, a quantitative research method was chosen with simulation as the tool to collect the required data to enable the analysis to be done comparatively.

\subsection{Data Collection}

Primary data was collected with the help of the OPNET 14.5 simulator. Secondary data was accessed by reviewing literature that was relevant to the study.

\subsection{Network Design}

In order to better study behavior and performance related issues of a network, the best approach is by way of simulation [20]. For the purposes of the study, two scenarios were created with the help of OPNET.

Scenario 1: In the first scenario, a network topology was configured with Frame Relay as the WAN with 6to4 tunneling as the transition mechanism. Traffic from FTP, Email and Database Query applications were sent over the Frame Relay WAN. The network performance parameters measured were Database Query response time, Application (Email and FTP) download time, End to End delay and Link utilization.

Scenario 2: In the second scenario, this time around, the WAN network was MPLS configured with Traffic Engineering in mind and again 6to4 transition mechanism was chosen. Traffic was provided by FTP, Email and Database query applications. The network performance parameters measured were Database Query response time, Application (Email and FTP) download time, End to End delay and Link utilization.

The network links, number of workstations (i.e. 50), routers and routing protocols remained same for both scenarios. Also, the simulation was carried out for the same duration for both scenarios simultaneously.

Figures 1 and 2 represent Frame Relay with 6to4 and MPLS with 6 to 4 respectively 


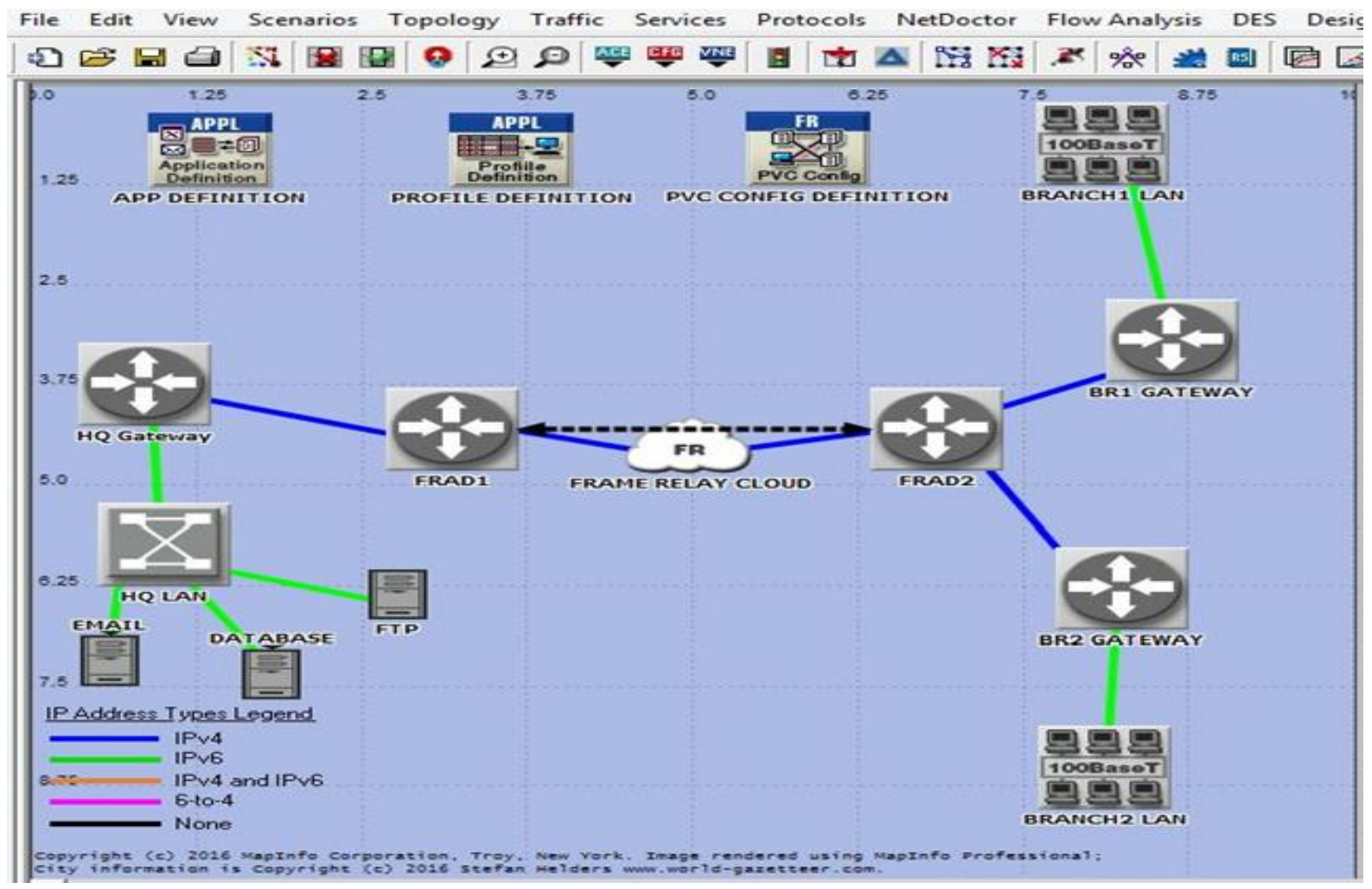

Figure 1. Network model for Frame Relay with 6to4 configuration

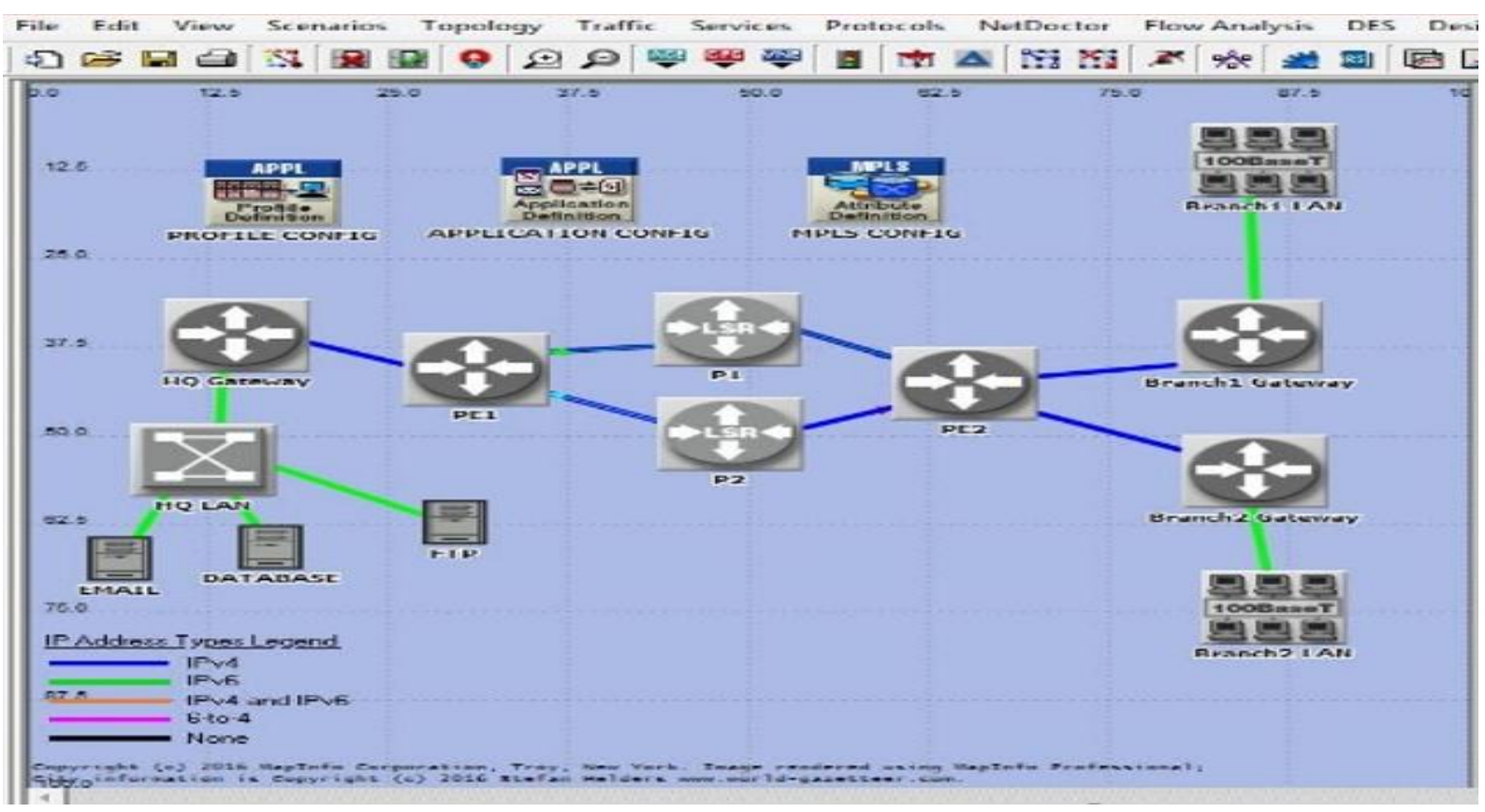

Figure 2. Network model for MPLS with 6to4 configuration

\section{RESULTS AND ANALYSIS}

The simulation was carried for the two scenarios namely MPLS6to4 and Frame_Relay6to4. For each scenario, a packet size of $1 \mathrm{MB}, 5 \mathrm{MB}$ and $10 \mathrm{MB}$ respectively representing low, medium and high packets were sent across the network with varying link speed (Switching speeds) of 500Mbps, 1Gbps and $1.5 \mathrm{Gbps}$ were set to see the performance effect on the network indexes selected. The simulation was carried out for nine consecutive times with an hour of simulation time for each run. The results were collected for the tenth and fiftieth minute. 
Table 1. Database Query Response Time with Packet Size of 5Mb

\begin{tabular}{|c|c|c|c|c|c|c|}
\hline \multirow{2}{*}{ SCENARIO } & \multicolumn{2}{|c|}{ 500Mbps } & \multicolumn{2}{c|}{ 1Gbps } & \multicolumn{2}{c|}{ 1.5Gbps } \\
\cline { 2 - 7 } & $\mathbf{1 0}$ MINS & $\mathbf{5 0}$ MINS & 10MINS & $\mathbf{5 0}$ MINS & 10 MINS & 50 MINS \\
\hline MPLS6to4 & 0.002588714 & 0.002588501 & 0.002584221 & 0.002584257 & 0.002582948 & 0.002582927 \\
\hline FR6to4 & 0.014065918 & 0.013874098 & 0.01403848 & 0.013854308 & 0.014033877 & 0.013846396 \\
\hline
\end{tabular}

Table 2. End to End Delay with Packet Size of $5 \mathrm{Mb}$

\begin{tabular}{|c|c|c|c|c|c|c|}
\hline \multirow{2}{*}{ SCENARIO } & \multicolumn{2}{|c|}{ 500Mbps } & \multicolumn{2}{c|}{ 1Gbps } & \multicolumn{2}{c|}{ 1.5Gbps } \\
\cline { 2 - 7 } & $\mathbf{1 0}$ MINS & $\mathbf{5 0}$ MINS & 10MINS & 50 MINS & 10 MINS & 50 MINS \\
\hline MPLS6to4 & 0.00004547152 & 0.00004722318 & 0.0000446843 & 0.00004669772 & 0.00004463987 & 0.000046439156 \\
& 1 & 4 & 41 & 7 & & \\
\hline FR6to4 & 0.000140542 & 0.000140629 & 0.000140202 & 0.000140309 & 0.000140066 & 0.000140200 \\
\hline
\end{tabular}

Table 3. Downlink Utilization with Packet Size of $5 \mathrm{Mb}$

\begin{tabular}{|c|c|c|c|c|c|c|}
\hline \multirow{2}{*}{ SCENARIO } & \multicolumn{2}{|c|}{ 500Mbps } & \multicolumn{2}{|c|}{ 1Gbps } & \multicolumn{2}{|c|}{ 1.5Gbps } \\
\hline & 10 MINS & 50 MINS & 10MINS & 50 MINS & 10 MINS & 50 MINS \\
\hline MPLS6to4 & 0.001621664 & 0.001475037 & 0.001504175 & 0.001459957 & 0.001465022 & 0.001442186 \\
\hline FR6to4 & 0.001520491 & 0.001430891 & 0.001434244 & 0.001433951 & 0.001459486 & 0.00145099 \\
\hline
\end{tabular}

\subsection{Graphical Representation of the Results}

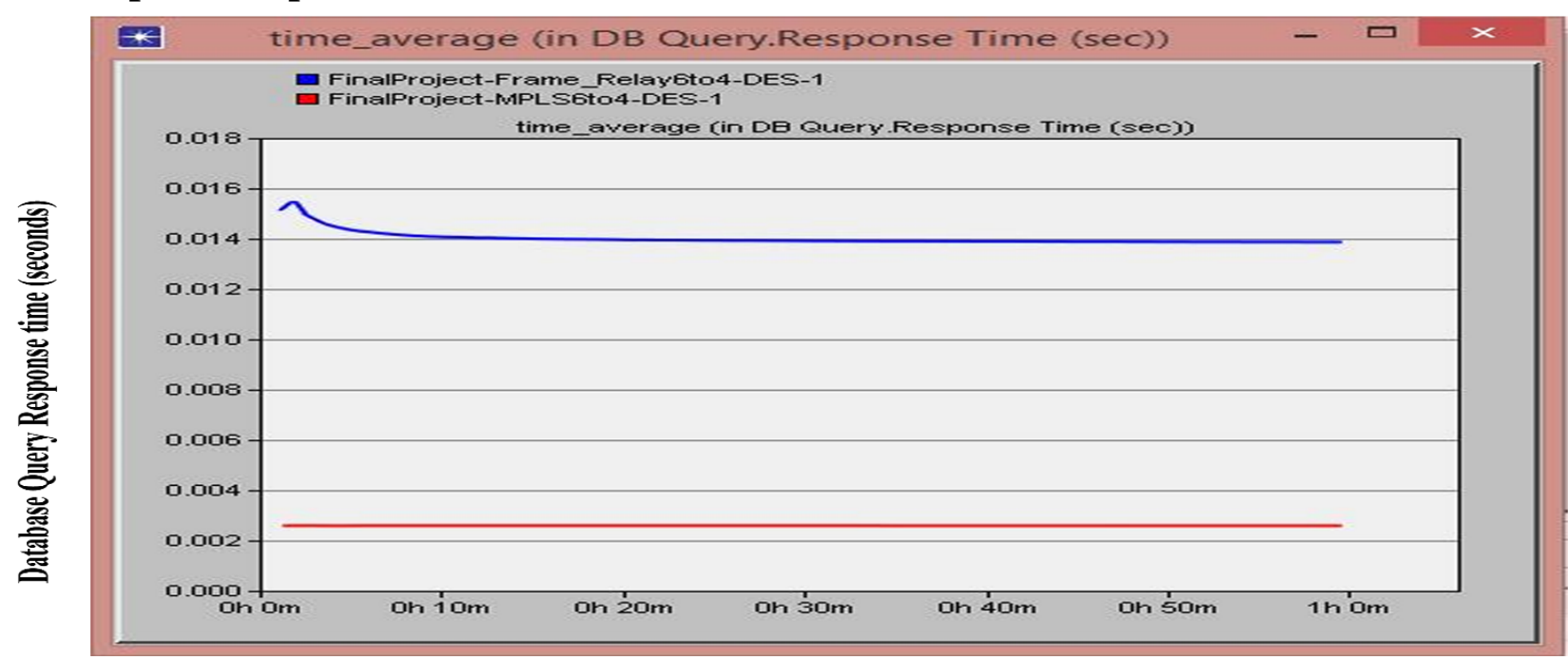

Simulation Time in Minutes

Figure 3. A Graph comparing Database Query Response Times for MPLS and Frame Relay using 6 to4

The database query response time is graphically presented in figure 3. In the scenario for frame relay with 6to4, the database query response time rises to its peak at a value of 0.015182278 during the first minute. This is so because 6to 4 mechanism has to encapsulate IPv6 packets inside IPv4 before transmission. There is a significant drop in the response time at the tenth minute with the value at 0.014033877 . This keeps dropping till the 30 th minute then a constant response time is attained until the end of the simulation.

The MPLS with 6to4 scenario on the other hand starts initially on the first minute with 0.002582635 rising to 0.002582948 in the tenth minute. MPLS builds Forwarding Information Base (FIB) over the period unlike frame relay which relies on DLCI 
for forwarding packets. The building of the FIB and the encapsulation of IPv6 packets in IPv4 causes the slight increase in response time. However, the response time becomes constant from the 20th minute till the end of the simulation.

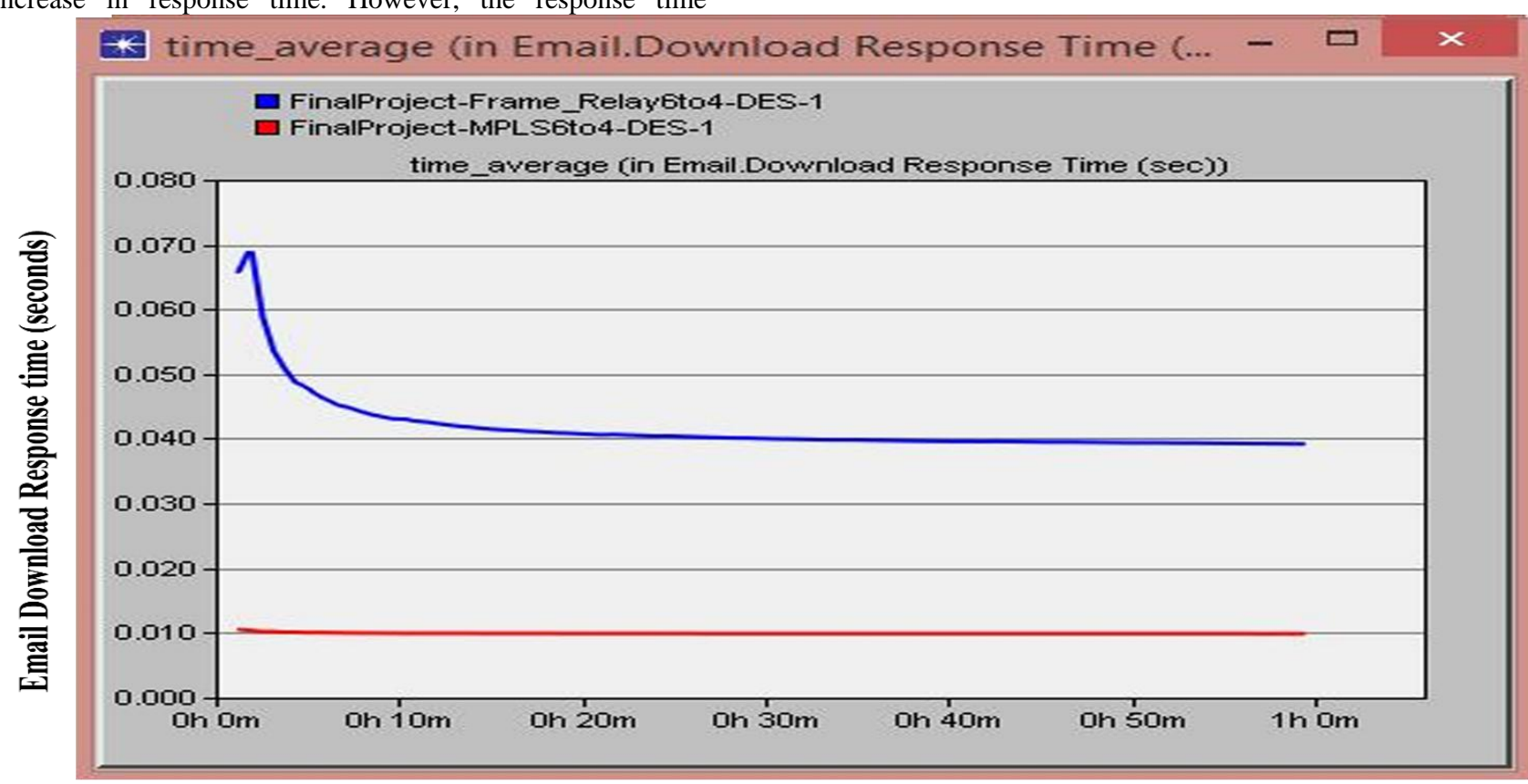

Simulation Time in Minutes

Figure 4. A Graph Comparing Email Download Response Times for MPLS and Frame Relay using 6 to4

Starting from a response time of 0.065705924 under the first minute for the frame relay 6 to 4 scenario. This figure drops to 0.042270792 on the tenth minute. The decline continues to about the 40th minute when a constant response time is achieved. This is observed in figure 4 .

The earlier rise in response time for email download can be attributed to de capsulation and encapsulation of IPv6 packets from IPv4 so as to enable smooth transition of the IPv6 packets over the IPv4 network. The other factor to aid the higher response time for email download on the frame relay scenario is the infrastructure which relies solely on DLCI to determine the destination of packets.

The MPLS with 6to4 scenario started under the first minute with 0.010513302 . This figure drops to 0.009942593 by the tenth minute as can be seen from the graph in figure 4.3. There is not so much activity on the graph for this scenario as can be observed in figure 4 , the changes in response time cannot be visually observed but a look at the data collected indicates subtle changes in response times collected. This goes to show that FIB together with T.E. employed by MPLS reduces response times relatively.

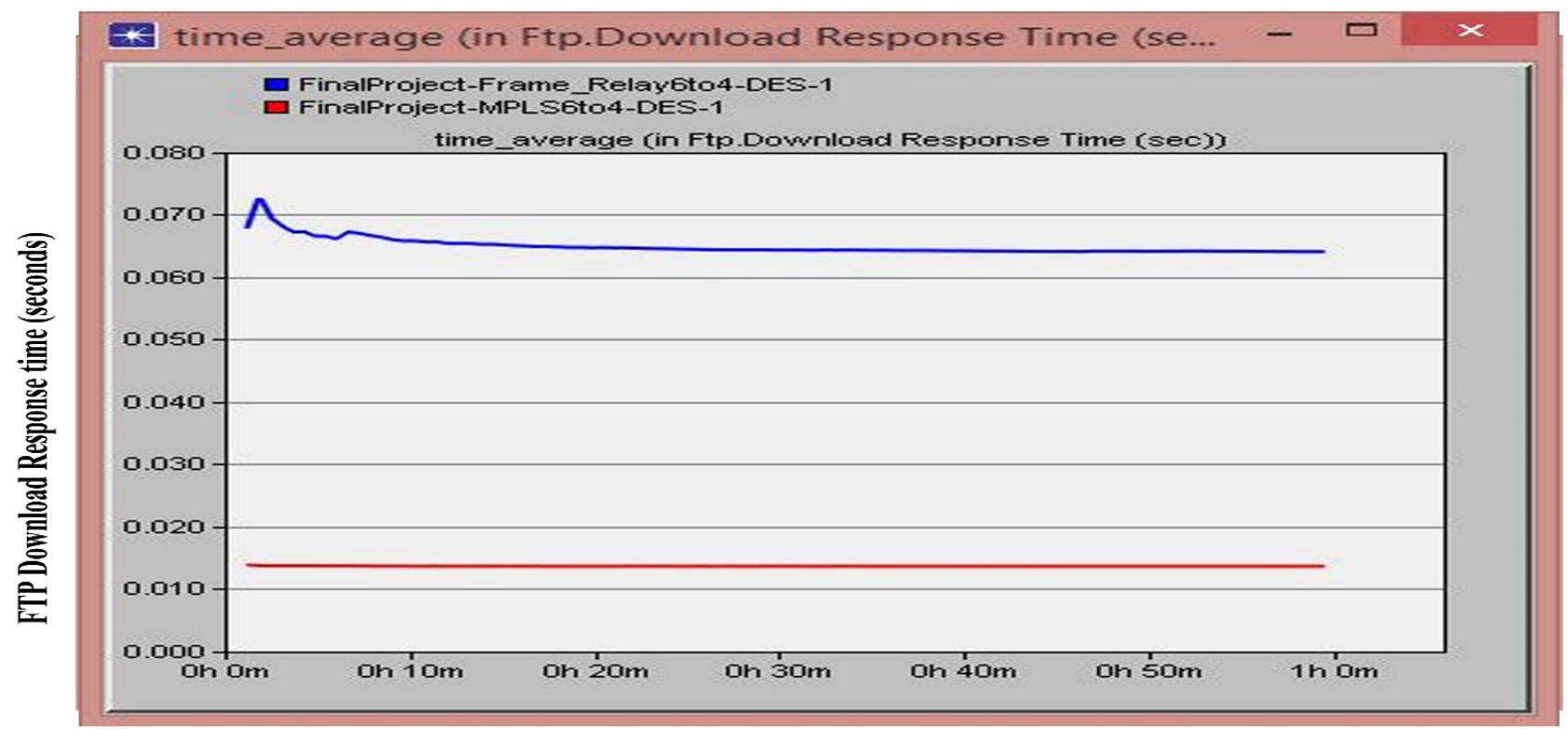

Simulation Time in Minutes

Figure 5. A Graph Comparing FTP Download Response Times for MPLS and Frame Relay using 6to4 
The 6 to 4 on Frame Relay hits its peak by the 3rd minute with a value of 0.06826725 and then declines gradually with a slight increase by the 10th minute and then declines again with a constant response time till the end of the simulation.

The MPLS with 6to4 scenario has a lower response time relative to that of Frame Relay. By inspection from the graph in figure 5, this observation can be made. For the first minute to the end of the simulation, there is not a significant change in response time. Comparatively, the MPLS scenario has a lower FTP download response time than Frame Relay due to factors mentioned earlier which includes the fact that MPLS employs Traffic Engineering (TE) and Quality of Service (QoS) strategies which aids the transmission of packets across the network which cannot be said about Frame Relay. Traffic Engineering ensures optimal traffic distribution and improves overall network utilization. It controls traffic and set up end to end routing path before forwarding data.

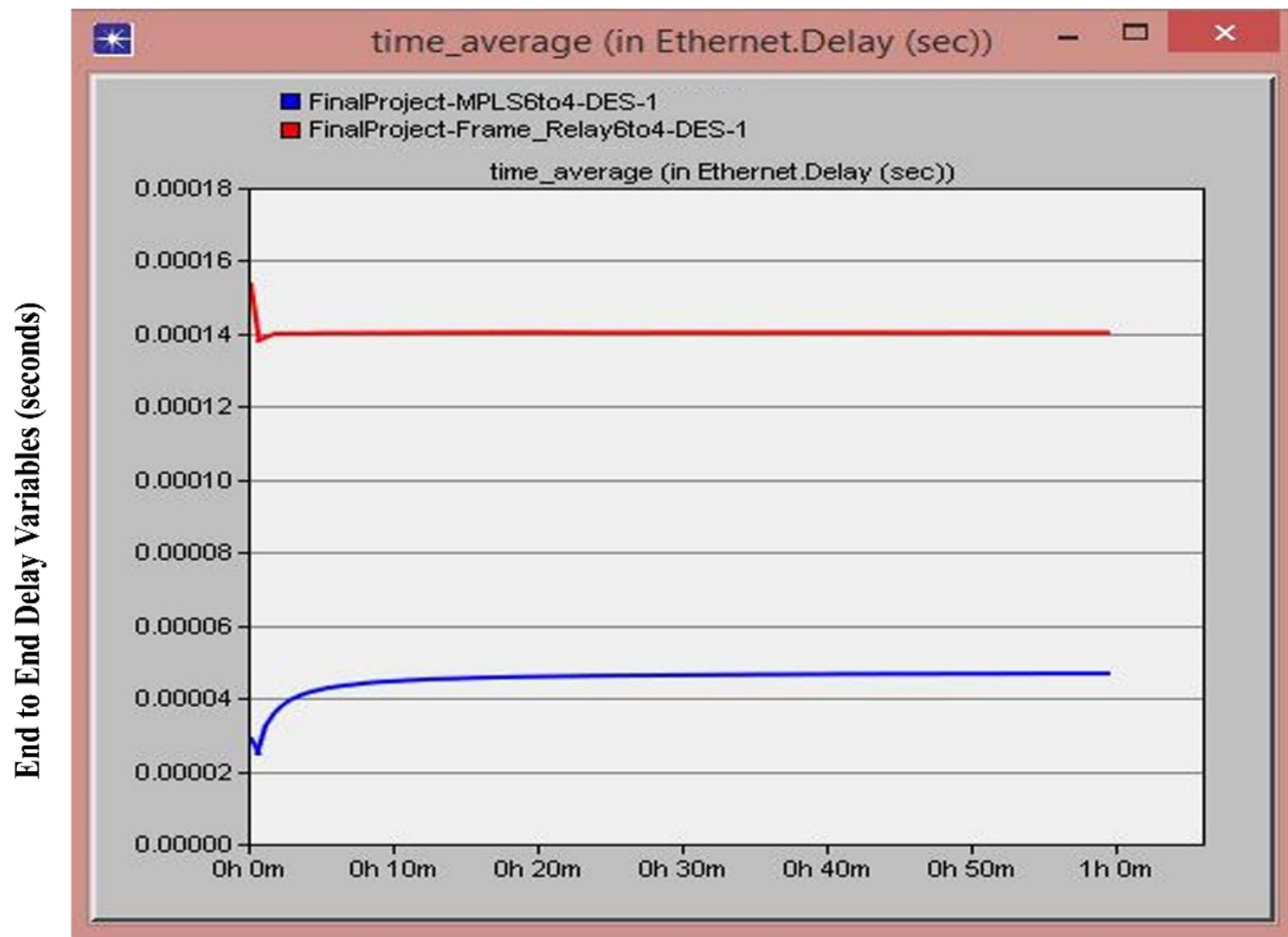

Simulation Time in Minutes

Figure 6. A Graph comparing End to End Delay for MPLS and Frame Relay using 6to4

The Frame Relay scenario with 6to4 has a higher end to end delay relative to that of MPLS. From the graph in figure 6, it can be observed that, the frame relay scenario with 6to4 starts with an end to end delay under the first minute with a value of 0.000153833 whereas that of MPLS with 6to4 under the same duration recorded an end to end delay of 0.000029398012 . By the end of the simulation, the respective scenarios recorded 0.000140198 and 0.000046538202 respectively for Frame Relay and MPLS scenarios with 6to4.
The end to end delay for the MPLS scenario is lower relative to that of Frame Relay because left to the 6to4 transition mechanism, the end to end delay value would have been higher but for the efficient management of network resources and flow of traffic, this figure is lower. MPLS forwarding technique is expedited because there is no need for the examination of packet header at every hop in a route to a destination hence reducing the delays. Frame Relay lacks these forwarding techniques and still forwards packets the conventional IP way. 


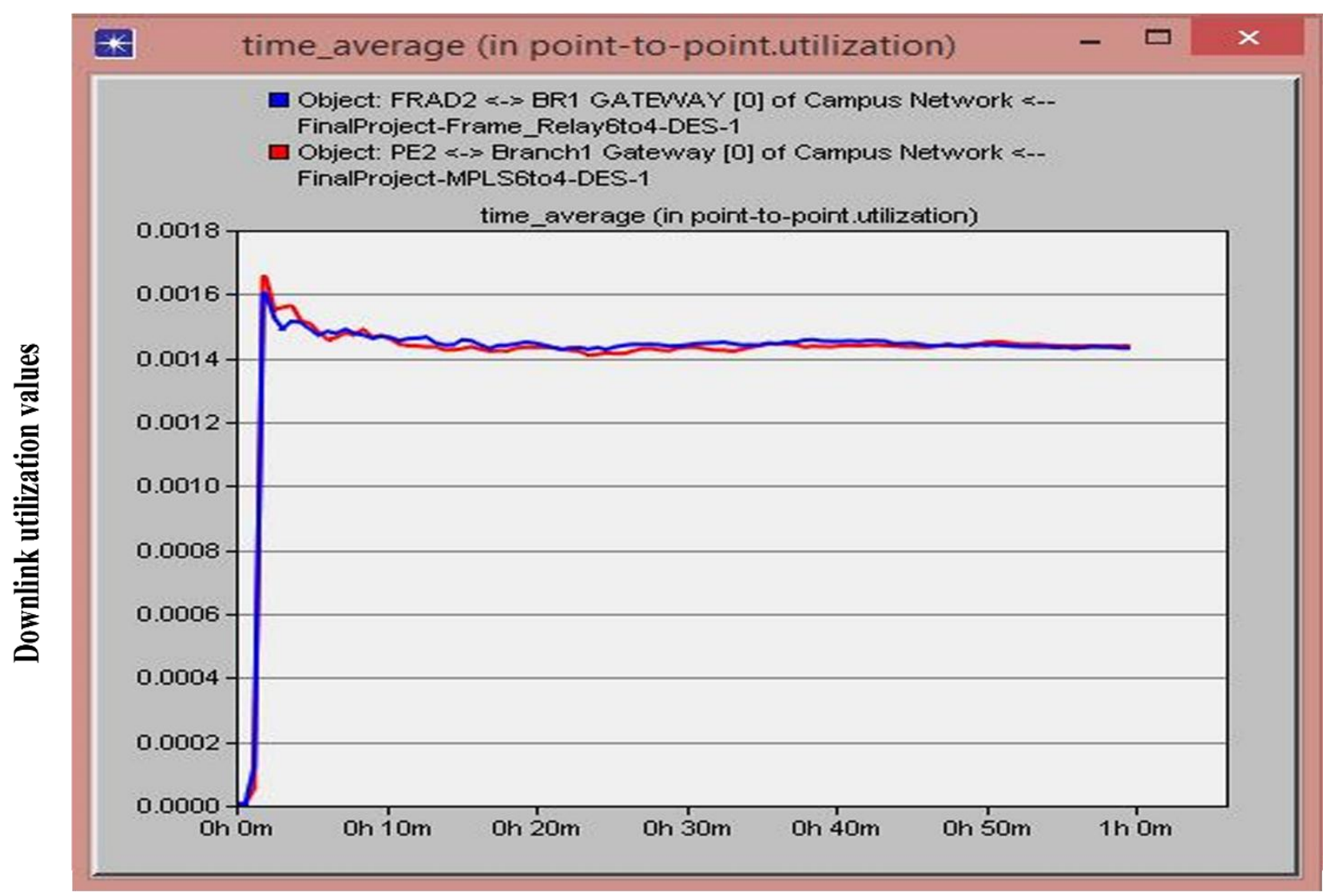

Simulation Time in Minutes

Figure 7. A Graph comparing Downlink Utilization for MPLS and Frame Relay using 6to4

In the 6to4 scenario with Frame Relay as the WAN, the story is no different. The time average graph presented in figure 7 indicates that the Frame Relay scenario was at its highest point under the second minute with a utilization value of 0.001657344 . This value keeps dropping slowly till the twentieth minute when the rate of decline is not so significant hovering around 0.001434 .

MPLS scenario peaks under the second minute with a value of 0.001604944 . This value is about 0.0000524 different from the Frame Relay scenario. This utilization value stabilizes around 0.001424 by the fortieth minute and continues till the end of the simulation.

The lower value for MPLS is attributed to the fact that MPLS has efficient ways of handling traffic differently so that they are not dropped and that the link is always utilized optimally. It also has features to as much as possible to avoid congestion. This feature is lacking in Frame Relay which relies on the conventional way of sending packets.

\section{CONCLUSION}

In conclusion, there is adequate evidence that IPv6 has advantage over IPv4 but to obtain a full IPv6 native network only is not achievable in the shortest time therefore the need for a transition period and transitional mechanisms. From the studies, 6to4 configured over MPLS is of great help in this transition as compared to 6to 4 configured on FR; the main objects under scrutiny. 6to 4 has its own added issues and delays as packets of IPv6 will have to be encapsulated in IPv4 packets, this time taken affects application response times which are not desirable. But MPLS by virtue of its approach in destination identification makes up for the lapses created by 6to4 unlike frame relay, has its strength in the reduction of cost in the medium used in transmission.

\section{RECOMMENDATION(S) \& FUTURE WORK}

MPLS is the much sought-after technology because of its ability to support multiple protocols at a go and also efficiently manage traffic. When presented with an option to choose between the 6to 4 running on either MPLS or FR, a combination of 6to 4 with MPLS is recommended in this transitional period and much research must be carried in this direction exploring the other opportunities and tweaks that can be made to make it more efficient.

In future, the performance of other transitional mechanisms will be studied when they are configured on FR and MPLS to see their output. To be precise, the dual stack as well as GRE mechanisms would be studied to see if they yield better results than 6 to 4 .

\section{REFERENCES}

[1] NRO (2011). Free pool of IPv4 address space depleted. Number Resource Organization Publications, Feb. 2011. [Online accessed 13-03-2020] http://www.nro.net/news/IPv4-free-pool-depleted.

[2] Graeme, C. (2014). Why Is The Transition To IPv6 Taking So Long? [Blog post]. Accessed on 13/03/2020 from http://teamarin.net/2014/08/13/transition-ipv6taking-long/

[3] Govil, J., \& N. Kaur. (2008). An examination of IPv4 and IPv6 networks: Constraints and various transition mechanisms. Southeastcon, IEEE. 
[4] Babar, A. K., Zardari, Z. A., Sirajuddin Qureshi, S. H., \& Hussaini, N. N. (2019). Assessment of IPv4 and IPv6 Networks with Different Modified Tunneling Techniques using OPNET. Assessment, 10(9).

[5] Suhail, A., Wajid A., H., \& Mohsin H., M. (2015). Performance Evaluation of IPv4 and IPv6 over MPLS Using OPNET. International Journal of Computer Applications, vol. 125, No.3, pp. 0975-8887.

[6] Grayeli, P., Sarkani, S., \& Mazzuchi, T. (2012). Performance analysis of IPv6 transition mechanisms over MPLS. International Journal of Communication Networks and Information Security, Vol.4, No.2.

[7] Rani, S., \& Vandana. (2015). Performance evaluation of IPV6 transition technologies. International Journal of Advanced Research in Computer Engineering \& Technology (IJARCET), Vol.4, Issue 6.

[8] Khannah, B., \& Alsadeh, A. (2017). Impact of IPv4/IPv6 Transition Techniques on Applications Performance.

[9] Albkerat, A., \& Issac, B. (2014). Analysis of IPV6 Transition Technologies. International journal of Computer Networks \& Communications, -. https://doi.org/10.5121/ijenc.2014.6502

[10] Georgescu, M., Hazeyama, H., Kadobayashi, Y., \& Yamaguchi, S. (2014). Empirical analysis of IPv6 transition technologies using the IPv6 Network Evaluation Testbed. In International Conference on Testbeds and Research Infrastructures (pp. 216-228). Springer, Cham.

[11] Wu, Y., \& Zhou, X. (2011). Research on the IPv6 performance analysis based on dual-protocol stack and tunnel transition. In 2011 6th International Conference on Computer Science \& Education (ICCSE) (pp. 10911093). IEEE

[12] Hossain, M. A.,Podder, D., Jahan, S., \& Hussain, M.(2016). Performance Analysis of Three Transition Mechanisms between IPv6 Network and IPv4 Network:
Dual Stack, Tunneling and Translation. International Journal of Computer (IJC). 20. 217-228.

[13] Kalwar, S., Bohra, N., \& Memon, A. A. (2015). A survey of transition mechanisms from IPv4 to IPv6-Simulated test bed and analysis. In 2015 Third International Conference on Digital Information, Networking, and Wireless Communications (DINWC) (pp. 30-34). IEEE.

[14] Singalar, S., \& Banakar, R. M. (2018). Performance analysis of IPv4 to IPv6 transition mechanisms. In 2018 Fourth International Conference on Computing Communication Control and Automation (ICCUBEA) (pp. 1-6). IEEE.

[15] Kim, P. S. (2017). Analysis and comparison of tunneling based IPv6 transition mechanisms. International Journal of Applied Engineering Research, 12(6), 894-897.

[16] Carpenter, B., \& Moore, K. (2001). Connection of IPv6 domains via IPv4 Clouds, Internet Engineering Taskforce RFC 3056. Retrieved from https://tools.ietf.org/html/rfc3056

[17] Wu, J., Cui, Y., Li, X., Xu, M., \& Metz, C. (2010) 4over6 transit solution using IP encapsulation and MPBGP extensions. IETF RFC5747. Retrieved from https://tools.ietf.org/html/rfc5747

[18] Nordmark, E., \& Gilligan, R. (2005). Basic transition mechanisms for IPv6 hosts and routers. RFC 4213, October. Retrieved from https://tools.ietf.org/html/rfc4213

[19] Cui, Y., Wu, J., Wu, P., Vautrin, O., \& Lee, Y. (2013). Public IPv4-over-IPv6 access network. IEEE RFC 7040 Retrieved from https://tools.ietf.org/html/rfc7040

[20] Okoh, K. E., Asante, M., \& Agbenyegah F., K. (2016). Investigating and Evaluating of Network Failures and Performance over Distributed WAN in Application Protocol Layer. International Journal of Scientific \& Technology Research, Vol. 5, Issue 01, ISSN 2277-8616, pp. 153-159. 